\title{
Effect of Perlite on Reducing Water Stress for Three Genotypes of Tomatoes Solanum lycopersicum L.
}

1. Vegetative growth

AL-Shammary, A. M. A. ${ }^{1}$; M. A. Abood ${ }^{2}$ and G. J. Hamdi ${ }^{1}$

${ }^{1}$ Prof. Dept. of Hort. and Landscape - College of Agric-Univ. of Diyala

${ }^{2}$ Assist. Prof.-Dept. of Soil and water Resources- College of Agric- Univ. of Diyala ghassanhamdi38@gmail.com

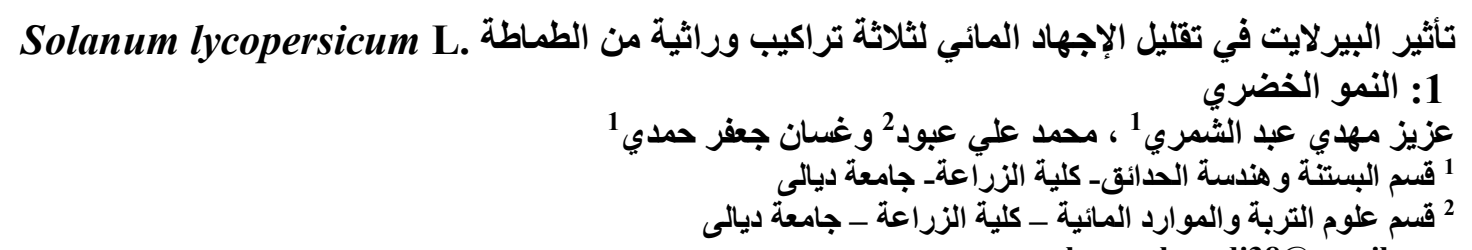
ghassanhamdi38@gmail.com

\section{الملخص}

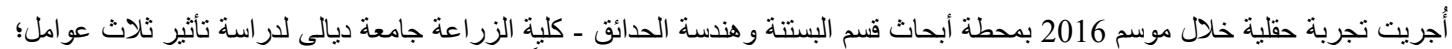

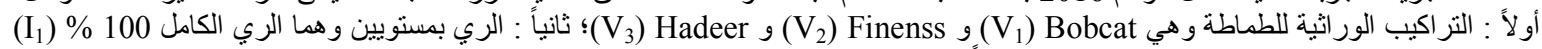

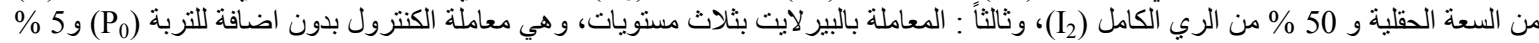

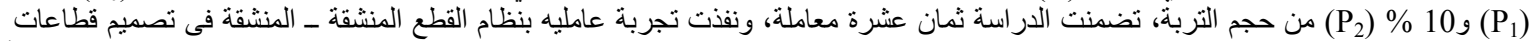

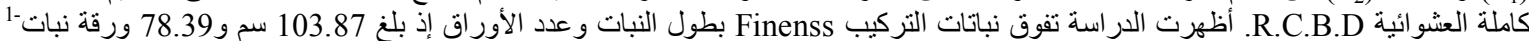

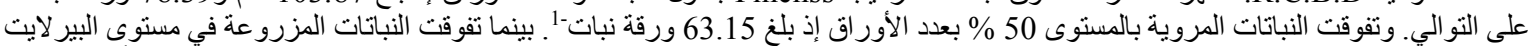

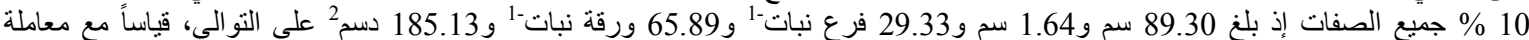

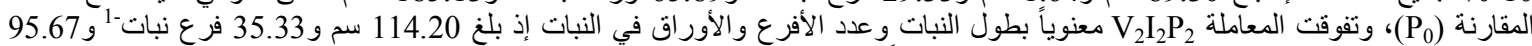

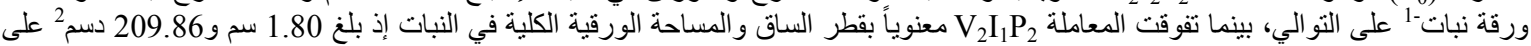

الكلمات المفتاحية: التراكيب الور اثية، البير لايت، الاجهاد المائي، الطماطة.

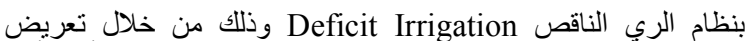

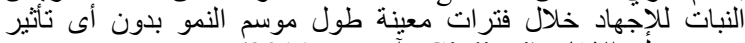

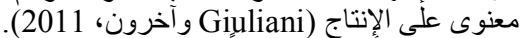

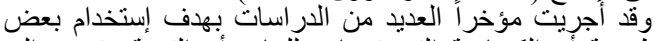

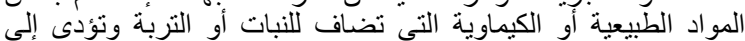

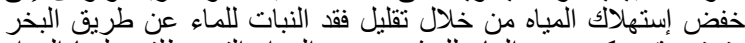

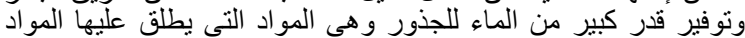

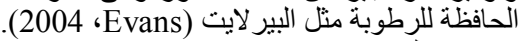

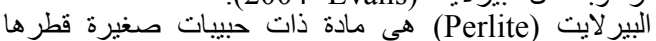

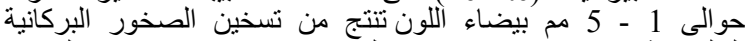

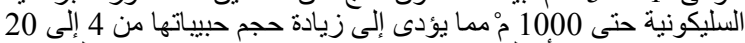

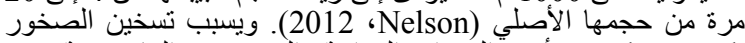

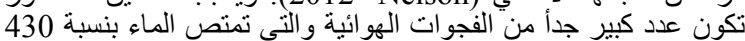

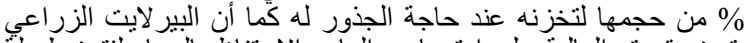

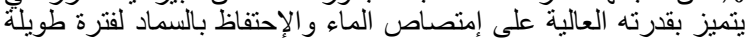

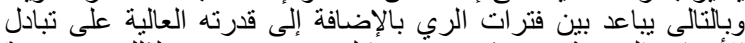

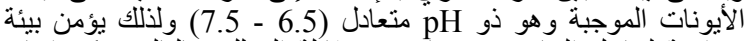

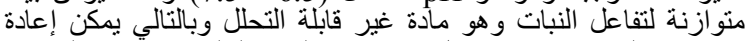

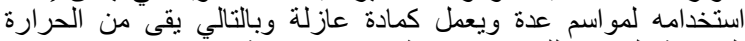

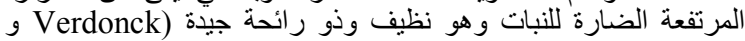

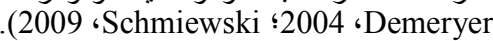
المواد وطر ائق العمل

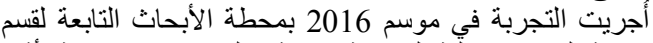

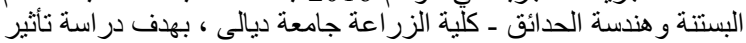

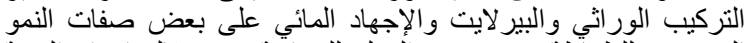

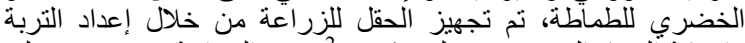

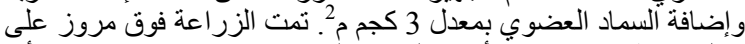

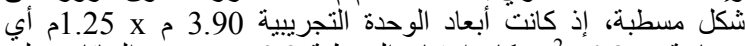

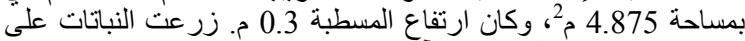

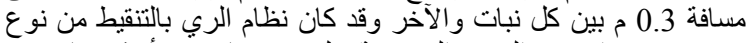
T- Tape

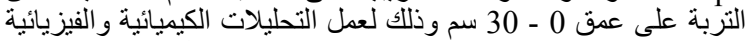

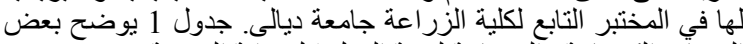
الصفات الكيميائية والفيزيائية لتربة الحقل قبل بداية التجربة.

\section{المقدمة}

الطماطة Tomato من محاصيل الخضر المهمة في العالم وهي

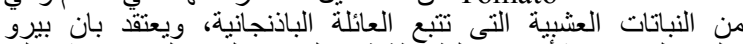

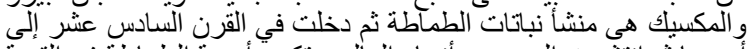

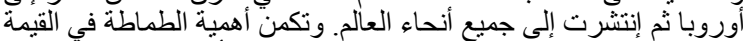

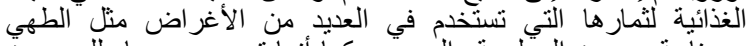

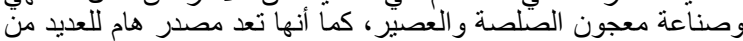

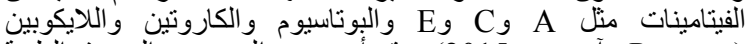
Perveen)

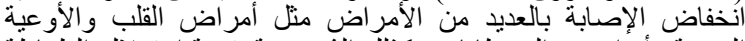

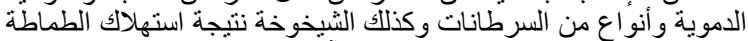

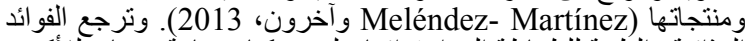

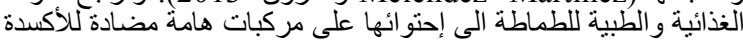

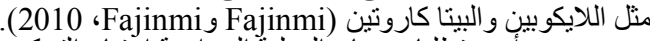

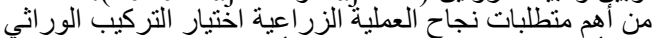

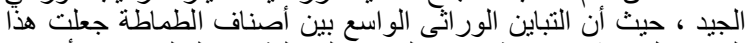

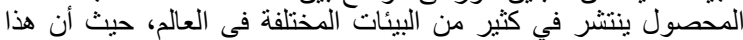

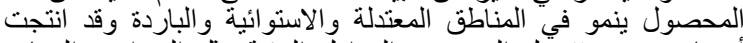

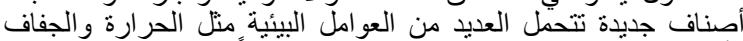

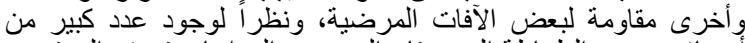

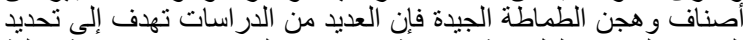

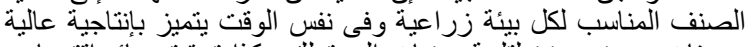
وصفات جودة مميزة لتلبية رغبات المستهلك وكذا تحقيق عائد إقتصادى التيل

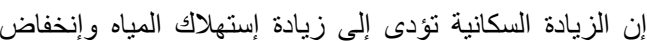

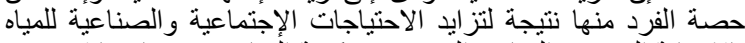

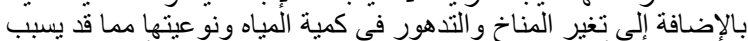

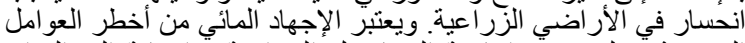

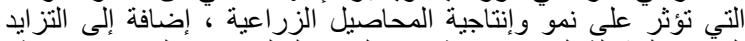

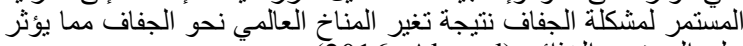

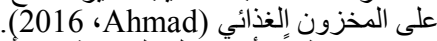
ونظر أ لأن مجال الزي الزراعة من أكبر المجالات المستهلكة للماء

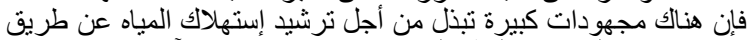

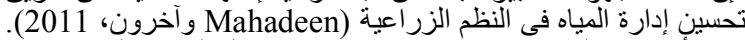
ومن أهم هذه الطرق هو استخدام استراتيجية بديلة لتوفير المياه تعرف 
AL-Shammary, A. M. A. et al.

3. عدد الأفرع الكلية (فرع نبات-1-).

تم أخذ عدد الآفرع الجانبية للخمسة نباتات السابقة /وحدة تجريبية في آخر الموسم ثم تم حساب المتوسط.

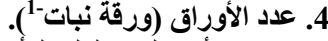

تم أخذ العدد الكلى للأور اق فى 5 نباتات عند نهاية الموسم ثم تم حساب المتوسط.

5. المساحة الورقية الكلية للنبات (دسمنبات-1).

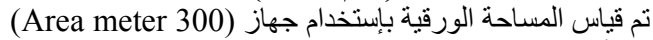

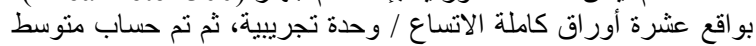

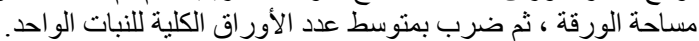
التصميم التجريبي و التحليل الإحصائي.

صمدت التجربة حسب نظأت

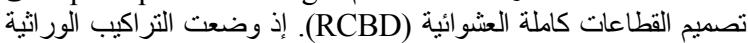

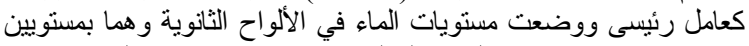

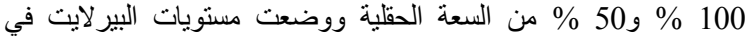
الوحدات تحت الثانوية بثلاث مستويات (صفر ،5 \% \% و 10 \% \%) ، تكونت

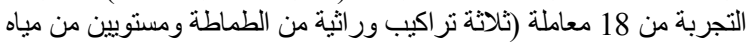

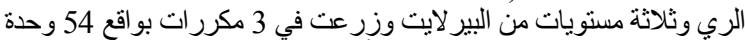

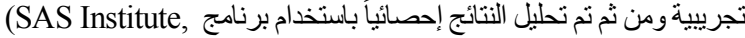
Inc., Cary, NC, USA, 2008) SAS

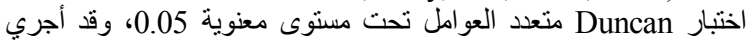

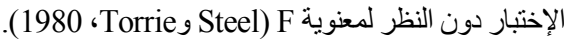

\section{النتائج والمناقشات}

أظهرت النتائج في جدول 2 أن طول النبات نأثر معنوياً نتيجة

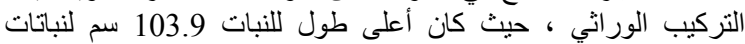

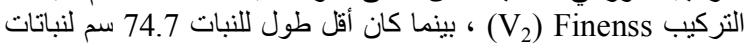

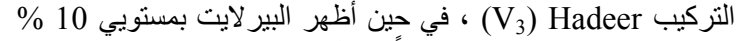
تفوق معنوياً لطول النبات مقارنة بمعاملة المقارنة

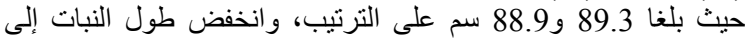

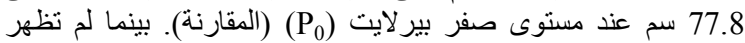
مستويات الري أي فروق معنوية في طول النبات

وكان هناك تأثير معنوي في في زيادة طول النيات النبات نتيجة للتداخل

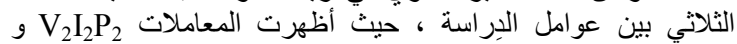
110.3 أعلى طول للنبات 114.2 V $11 \mathrm{I}_{1} \mathrm{P}_{2}, \mathrm{~V}_{2} \mathrm{I}_{2} \mathrm{P}_{1}, \mathrm{~V}_{2} \mathrm{I}_{1} \mathrm{P}_{1}$ و107.0 و103.2 سم على التوالي، في حين انخفض إلى 62.6 سم في للفي المعاملة 2.قطر الساق (سم). أظهرت النتائج أن صفة قطر الساق تأثرت معنوياً بالتراكيب

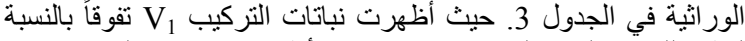

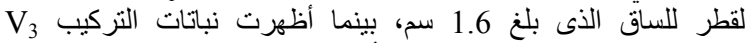

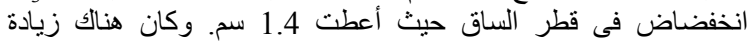

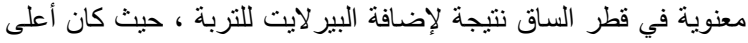

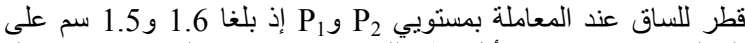

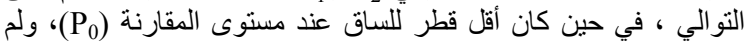

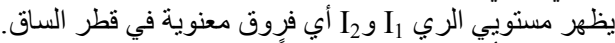

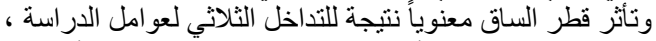

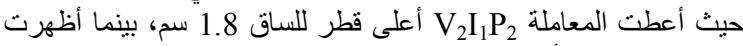
المعاملة 3. عدد الأفرع الكلية (فرع نبات ألمات).

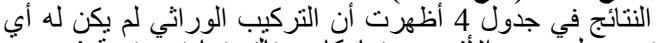

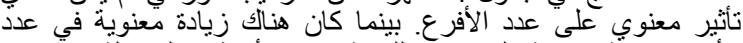

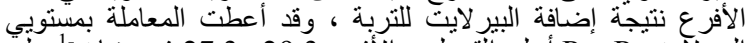

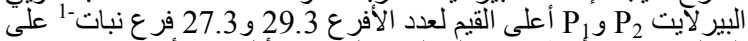

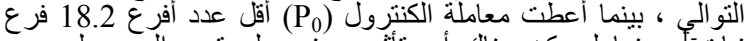

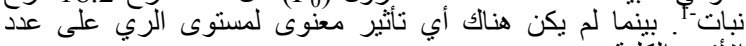

وكَان هنالك تأثثير معنوي على عدد الأفرع نتيجة للتداخل الثلاثي الأفرع الكلية

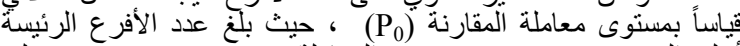

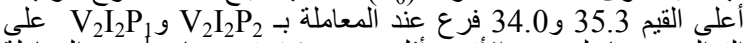
التوالي، بينما بلغ عدد الأفرع أقل عدد 14.7 فرع نبات- عند المعاملة
جدول 1. الصفات الكيميائية والفيزيائية للتربة.

\begin{tabular}{|c|c|c|c|}
\hline القيمة & الوحدة الوحة & & صفات التربة \\
\hline 7.04 & ---- & & الحموضة pH \\
\hline 7.55 & Ds $m^{-1}$ & & التوصيل الكهربي (1:1) \\
\hline 54.01 & & $\mathrm{~N}$ & \\
\hline 8.04 & ملغم كغم-1 - مل & $\mathrm{P}$ & العناصر المتاحة \\
\hline 81.79 & & $\mathrm{~K}$ & \\
\hline 6.90 & & & المادة العضوية \\
\hline $\begin{array}{l}260.10 \\
286.60\end{array}$ & غم كغم-1 & 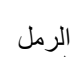 & $\mathrm{CaCO}_{3}$ \\
\hline $\begin{array}{l}591.20 \\
122.20\end{array}$ & & الغرين & معزولات التربة \\
\hline Silty loam & مزيجة غرينية & & ق قو ام التزبة \\
\hline 25 & $\%$ & & السعة الحقلية \\
\hline 1.35 & غم سم-3 & & الكثافة الظاهرية \\
\hline
\end{tabular}

وقد إثتملت الاراسة العوامل التالية :

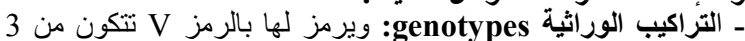
تر اكيب ور اثية هي bobcat

ـ معدلات الرى : ويرمز لها بالرمز I و هى عبارة عن مستويان من الري

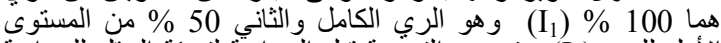

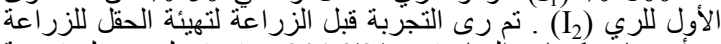

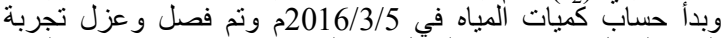

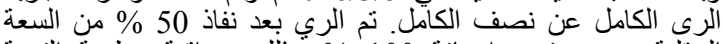

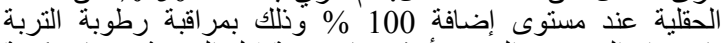

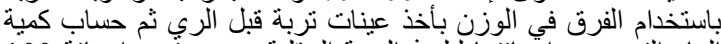

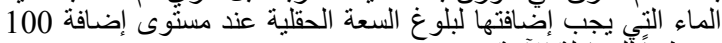
\% مبقاً لَّمعادلة ألآتية :

$$
\mathbf{d}=\boldsymbol{\theta}_{\mathrm{v}} \times \mathbf{D}
$$

d عمق الماء الذي تمت إضافته (سم) d

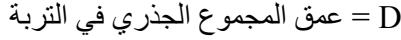

Ov

$$
\boldsymbol{\Theta}_{\mathrm{v}}=\boldsymbol{\Theta}_{\mathrm{m}} \times \boldsymbol{\rho}_{\mathrm{b}}
$$

= Om

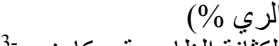

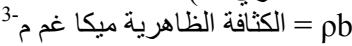

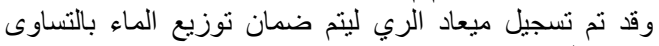

لجميع الوحدات التجريبية. 1. السعة الحقلية + نقطة الذبول.

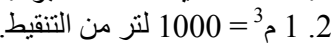

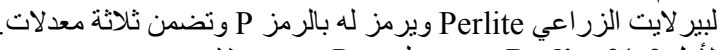

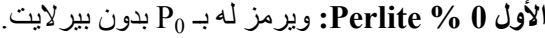

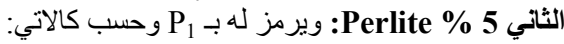

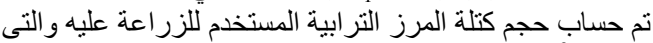

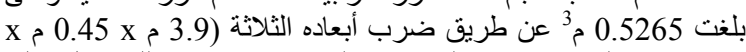

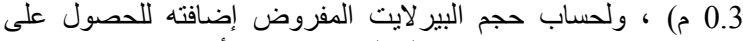

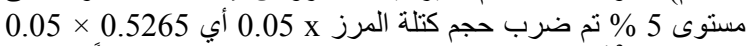

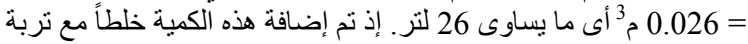
المرز لكل وحدة تجريبية.

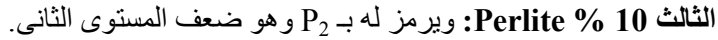
زرعت بذور التراكيب الور اثية للطماطة فى نهاية شهر ينائ

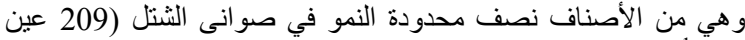

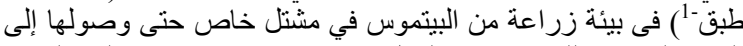

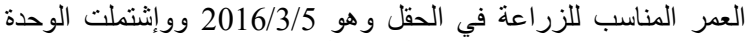
على 13 نبات مع ترك نبات فاصل بلين كل فئ وحدنين.

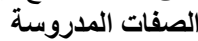

تم أخذ البيانات من النباتات تحت الدراسة وذلك بهدف تقدير

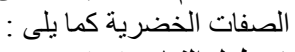

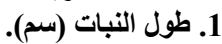

نم قياس طول النبات فى 5 نباتات عند نهاية الموسم باستخدام

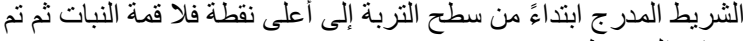

حساب المتوسط (IPGRI, 1996).

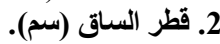

تم قياسه فى الخمسة نباتات السابقة عند مستوى سطح التربة باستخدام القدمة قدئ Vernier ثم تم حساب المتوسط. 
جدول 4. تأثير التراكيب الوراثية ومستوى البيرلايت والإجهاد المائي

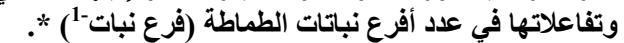

\begin{tabular}{|c|c|c|c|c|c|}
\hline التفاعل & \multicolumn{3}{|c|}{ مستويات البيرلايت } & \multirow{2}{*}{ معدلات } & \multirow{2}{*}{ لوراثية لتلمة } \\
\hline $\mathbf{V} \times \mathbf{I}$ & $\mathbf{P}_{2}$ & $\mathbf{P}_{1}$ & $\mathbf{P}_{0}$ & & \\
\hline 26.0 & 30.0 & 29.0 & 19.0 & \multirow{2}{*}{$\mathrm{I}_{1}$} & \\
\hline $\mathrm{a}$ & $a b c$ & $a b c$ & bed & \multirow{3}{*}{\multicolumn{2}{|c|}{$\mathrm{I}_{2}$}} \\
\hline 26.4 & 31.3 & 28.0 & 20.0 & & \\
\hline $\mathrm{a}^{\mathrm{a}}$ & $a b$ & abc & bcd & & \\
\hline $\begin{array}{c}23.8 \\
\mathrm{a}\end{array}$ & $\begin{array}{c}26.3 \\
\text { a-d }\end{array}$ & $\begin{array}{c}25.7 \\
\text { a-d }\end{array}$ & $\begin{array}{l}19.3 \\
\text { bcd }\end{array}$ & \multicolumn{2}{|l|}{$\mathrm{I}_{1}$} \\
\hline 29.2 & 35.3 & 34.0 & 18.3 & \multirow{2}{*}{\multicolumn{2}{|c|}{$\mathrm{I}_{2}$}} \\
\hline 22.0 & $\stackrel{\mathrm{a}}{26.7}$ & $\stackrel{\mathrm{a}}{24}$ & $\begin{array}{l}\text { bcd } \\
147\end{array}$ & & \\
\hline $\mathrm{a}$ & $a-d$ & a-d & d & \multicolumn{2}{|l|}{$\mathrm{I}_{1}$} \\
\hline 22.2 & 26.3 & 22.7 & 17.7 & \multirow{3}{*}{\multicolumn{2}{|c|}{$\mathrm{I}_{2}$}} \\
\hline $\mathrm{a}$ & $\begin{array}{l}\mathrm{a}-\mathrm{d} \\
29.3\end{array}$ & $\begin{array}{l}\mathrm{a}-\mathrm{d} \\
27.3\end{array}$ & $\begin{array}{c}\mathrm{cd} \\
18.2\end{array}$ & & \\
\hline & A & A & $\mathrm{B}$ & & \\
\hline \multicolumn{6}{|c|}{ 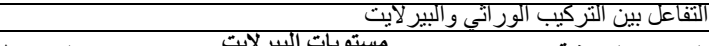 } \\
\hline المتوسط & \multicolumn{3}{|c|}{ مستويات البيرلايت } & \multicolumn{2}{|c|}{ لتراكيب الوراثية } \\
\hline 26.2 & $\frac{12}{30.7}$ & $\frac{1}{28.5}$ & $\frac{\mathbf{1} 0}{19.5}$ & & \\
\hline A & $\mathrm{a}$ & $\mathrm{a}$ & bc & & \\
\hline 26.5 & 30.8 & 29.8 & 18.8 & & \\
\hline A & $\mathrm{a}$ & $\mathrm{a}$ & bc & & \\
\hline 22.1 & 26.5 & 23.7 & 16.2 & & \\
\hline A & \multirow{2}{*}{\multicolumn{5}{|c|}{ التفاعل بين معدلات الري و البير لايت }} \\
\hline & & & & & \\
\hline المتوسط & \multicolumn{3}{|c|}{ مستويات البيزلايت } & \multirow{2}{*}{\multicolumn{2}{|c|}{ معلات الري }} \\
\hline r & $\mathbf{P}_{2}$ & $\mathbf{P}_{1}$ & $\mathbf{P}_{0}$ & & \\
\hline 23.9 & 27.7 & 26.5 & 17.7 & & \\
\hline A 259 & a & $\underset{28}{\mathrm{a}}$ & $\begin{array}{c}\mathrm{b} \\
187\end{array}$ & & \\
\hline A & $\mathrm{a}$ & $\mathrm{a}$ & $\mathrm{b}$ & & \\
\hline
\end{tabular}

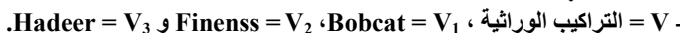

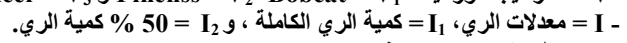

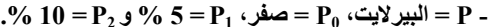

- هناك فروق مغنوية بين المتوسطات التي تحمل حروفاً مختلفة عذد مستوى احتمال 0.05 للعوامل الرئيسة والتفاعلات بينها.

- معنوية متوسطات التأثيرات الرئيسية يثار إليها بالأحرف الكبيرة بينما معنوية متوسطات التذاخلات يشار إليها بالأحرف الصغيرة. 4. عدد الأوراق (ورقة نبات-1).

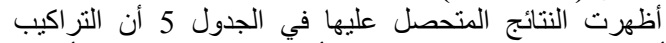

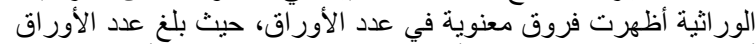

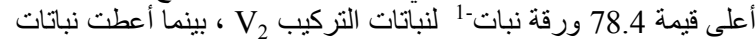

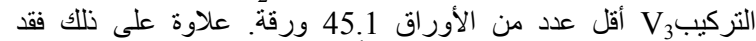

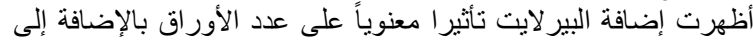

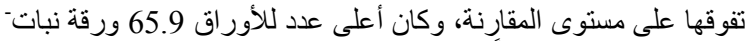

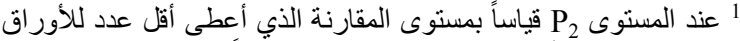

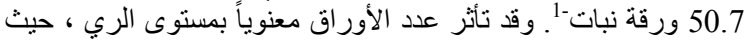

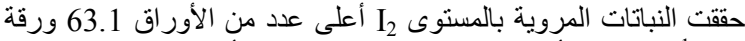

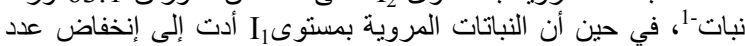

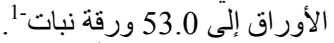

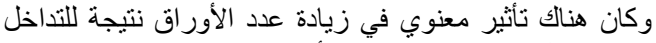

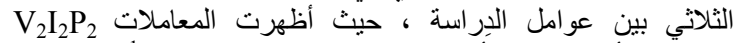
و أعلى عدد للأوراق 95.7 و و90.7 بينما انخفض إلى 36.0 ورقة نبات الميات في المعاملة

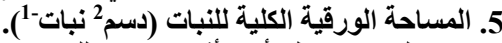

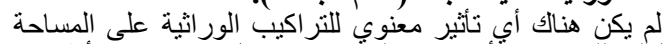

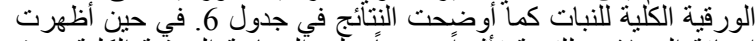

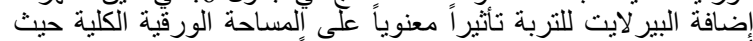

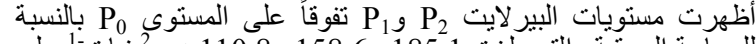

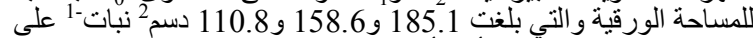

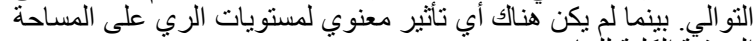

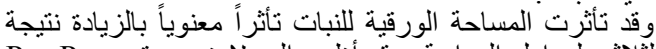

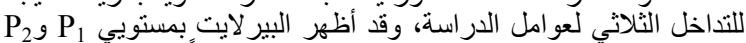

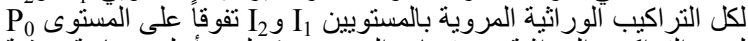

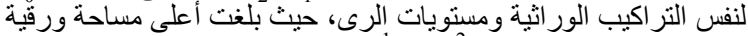
القيم 209.9 و 201.1 دسم² نبات على التو الي، بينما أدت المعاملة Vل

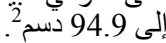

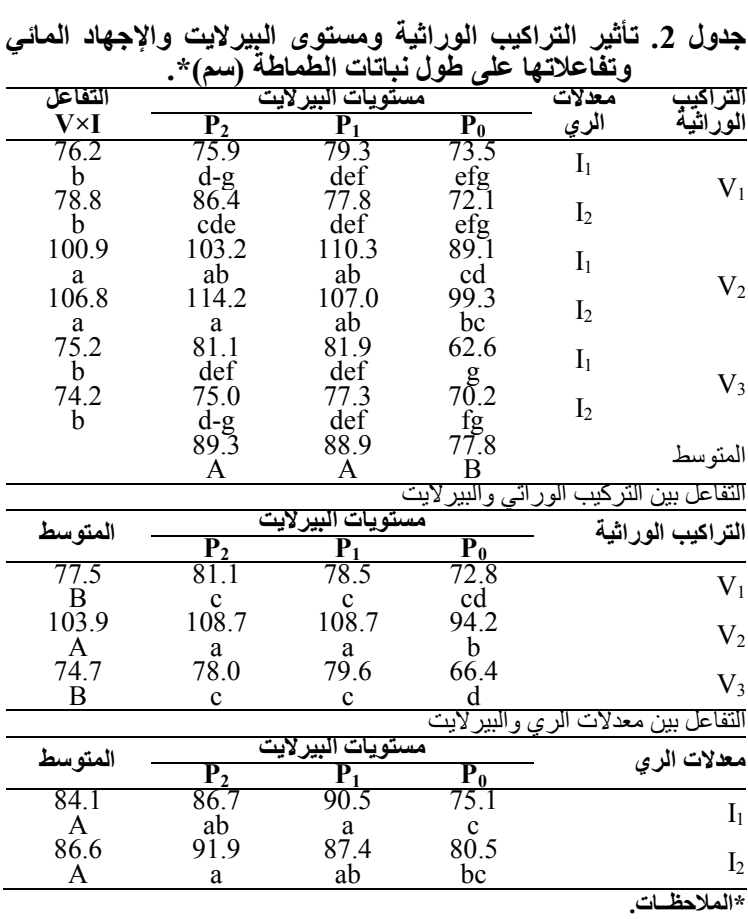

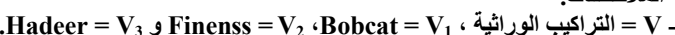

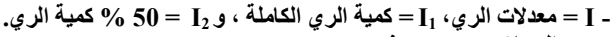

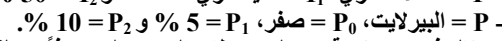
- هناك فروق معنوية بين المتوسطات التي تحمل حروفاً مختلفة عند مستوى احتمال 0.05 للعوامل الرئيسة والتفاعلات بينها.

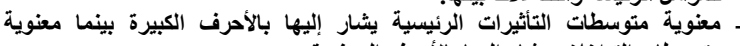
متوسطات التذاخلات يثار إليها بالأحرف الصغيرة

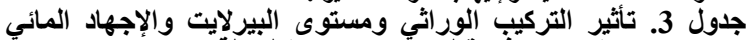

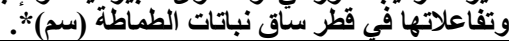

\begin{tabular}{|c|c|c|c|c|c|}
\hline التئاعل & \multicolumn{3}{|c|}{ مستويات البيرلايت } & \multirow{2}{*}{ معدلات } & \multirow{2}{*}{ الكراثيَّة } \\
\hline $\mathbf{V} \times \mathbf{I}$ & $\mathbf{P}_{2}$ & $\mathbf{P}_{1}$ & $\mathbf{P}_{0}$ & & \\
\hline 1.5 & 1.7 & 1.5 & 1.3 & $\mathrm{I}_{1}$ & \\
\hline $\begin{array}{l}\mathrm{b} \\
1.6\end{array}$ & $\begin{array}{l}\mathrm{ab} \\
1.7\end{array}$ & $\begin{array}{l}\mathrm{bc} \\
1.6\end{array}$ & ${ }_{1.5}^{d}$ & & $\mathrm{~V}$ \\
\hline$a b$ & $\mathrm{ab}$ & $\mathrm{b}$ & bc & $\mathrm{I}_{2}$ & \\
\hline 1.6 & 1.8 & 1.7 & 1.4 & $\mathrm{I}_{1}$ & \\
\hline $\mathrm{a}$ & $\mathrm{a}$ & $\mathrm{ab}$ & $\mathrm{cd}$ & & $\mathrm{V}$ \\
\hline $\begin{array}{c}1.4 \\
\mathrm{c}\end{array}$ & $\begin{array}{l}1.4 \\
\text { cd }\end{array}$ & $\begin{array}{l}1.5 \\
\mathrm{bc}\end{array}$ & $\begin{array}{c}1.3 \\
\mathrm{e}\end{array}$ & $\mathrm{I}_{2}$ & \\
\hline $\begin{array}{l}1.4 \\
b c\end{array}$ & 1.6 & 1.4 & 1.3 & $\mathrm{I}_{1}$ & \\
\hline 1.4 & 1.6 & 1.4 & 1.3 & $I_{2}$ & \\
\hline $\mathrm{bc}$ & $\mathrm{b}$ & $\mathrm{cd}$ & $\mathrm{d}$ & $1_{2}$ & \\
\hline & 1.6 & 1.5 & $\begin{array}{l}1.3 \\
B\end{array}$ & & 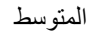 \\
\hline & \multicolumn{5}{|c|}{ التنفاعل بين التركيب الو راتي و البير لايت } \\
\hline المته سط & \multicolumn{3}{|c|}{ المستويات البيرلايت } & \multirow{2}{*}{\multicolumn{2}{|c|}{ التراكيب الوراثية }} \\
\hline الموسط & $\mathbf{P}_{2}$ & $\mathbf{P}_{1}$ & $\mathbf{P}_{0}$ & & \\
\hline 1.6 & 1.7 & 1.6 & 1.4 & & \\
\hline A & a & abc & $\mathrm{cd}$ & & \\
\hline 1.5 & 1.6 & 1.6 & 1.3 & & \\
\hline A & abc & $\mathrm{ab}$ & $\mathrm{d}$ & & \\
\hline 1.4 & 1.6 & 1.4 & 1.3 & & \\
\hline $\mathrm{B}$ & \multirow{2}{*}{\multicolumn{5}{|c|}{ التفاعل بين معدلات الري و البيرلايت $\quad$ ل }} \\
\hline & \multicolumn{2}{|c|}{ إل مستويات البيرلايت } & & & \\
\hline 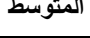 & $\mathbf{P}_{2}$ & $\mathbf{P}_{1}$ & $\mathbf{P}_{0}$ & & 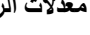 \\
\hline 1.5 & 1.7 & 1.6 & 1.3 & & \\
\hline A & $\mathrm{a}$ & $a b$ & $\mathrm{~d}$ & & \\
\hline 1.5 & 1.6 & 1.5 & 1.4 & & \\
\hline A & $a b$ & $\mathrm{bc}$ & $\mathrm{cd}$ & & \\
\hline
\end{tabular}

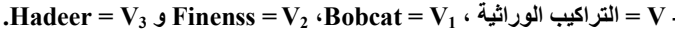

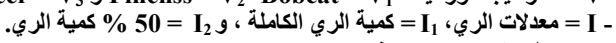

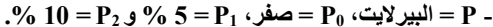
ـ ـ هناك فروق مغنوية بين المتوسطات التي تحمل حروفاً مختلفة عند مستوى احتمال 0.05 للعوامل الرئيسة والتفاعلات بينها. - معنوية متوسطات التأثيرات الرئيسية يثار إليها بالأحرف الكبيرة بينما معنوية متوسطات التذاخلات يشار إليها بالأحرف الصغيرة الصئة 
إن تباين التراكيب الوراثية في تركيبها الجيني هو السبب

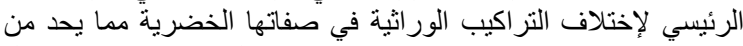

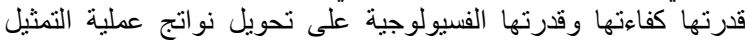

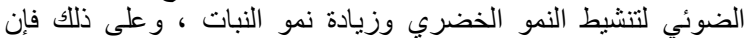

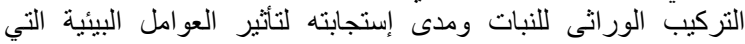

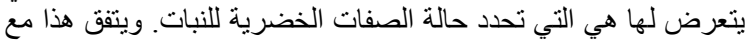
ماوجده Mehadi وآخرون (2016) و Challa وآخرون (2017).

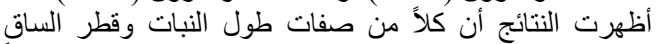

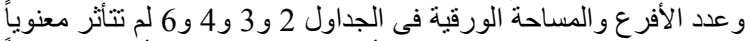

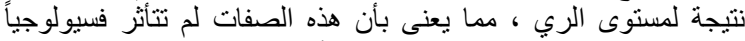

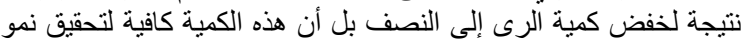

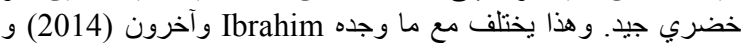
Etissa

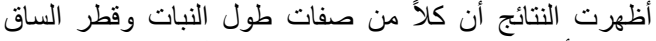

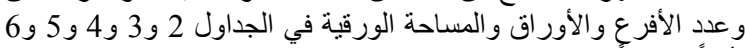

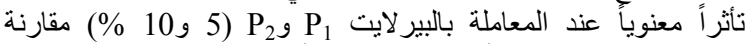

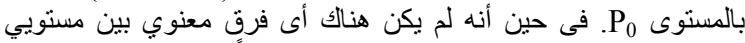

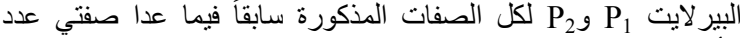
الأور اق و المساحة الورقية.

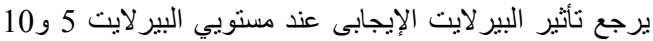

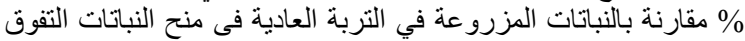

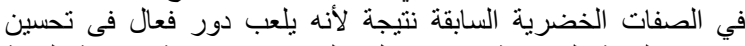

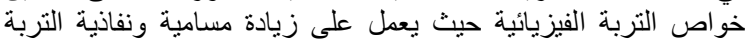

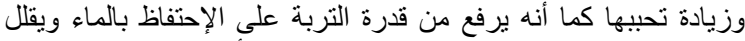

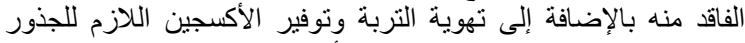

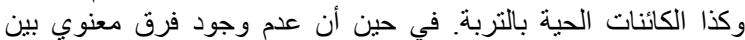

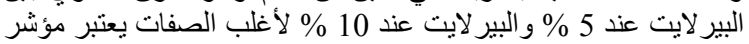

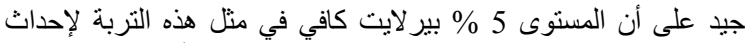

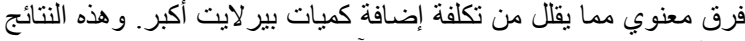

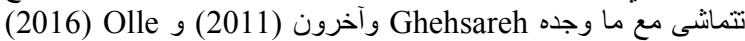
و Sedaghat وآخرون (2017) و Garameshwarareddy و و و

(2017) وتختلف عما وجده Haddad) (2007) (2017)

$$
\text { المراجع - (المبع }
$$

Ahmad, P. 2016. Water Stress and Crop Plants. A Sustainable Approach, Vol 2. department of Botany, S.P. College, Srinagar, Jammu and Kashmir, India.

Bhati, V. 2017. Evaluation of tomato genotypes for growth, yield and quality traits under foothills condition of Nagaland, India. Int. J. Curr. Microbiol. App. Sci. 6(3): 1645-1649.

Buhroy, S. ; T. Arumugam ; P. Irene Vethamoni ; N. Manivannan and P. Jeyakumar. 2017. Effectiveness of drought tolerance indices to identify tolerant genotypes with high yielding potential in tomato (Solanum lycopersicum L.). Int. J. Curr. Microbiol. App. Sci. 6(4): 20932103.

Challa, A. ; Z. Shelemew and A. Ambonsa. 2017. Evaluation of low cost drip irrigation technology through tomato production: in adami tulu jidokombolcha district, mid-rift valley of ethiopia. International Journal of Natural Resource Ecology and Management. 2(2): 32-37.

Etissa, E. ; D. Nigussie ; A. Tena ; A. Yibekal, and D. Lemma. 2014. Growth and physiological response of tomato to various irrigation regimes and integrated nutrient management practices. African Journal of Agricultural Research. 9(19):1484-1494.

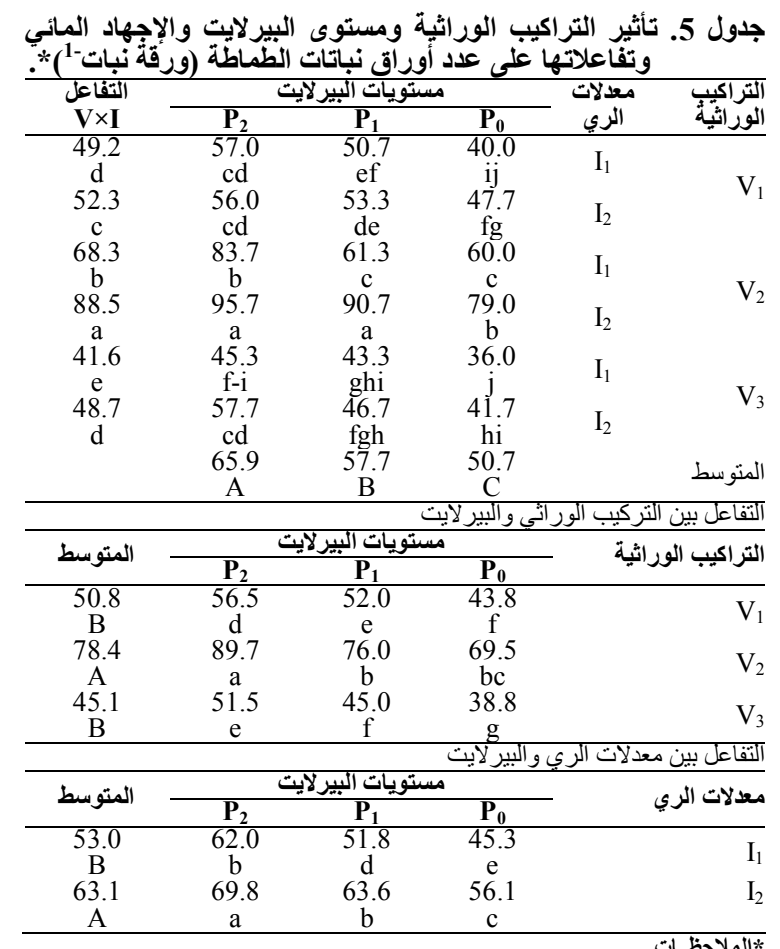

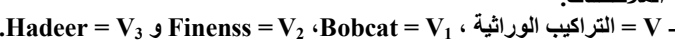

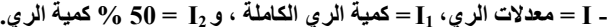

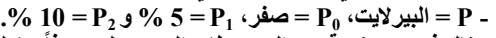
ـ هناك فروق مغنوية بين المتوسطات التي تحمل حروفاً مختلفة عند مستوى احتمال 0.05 لـ اللعو امل الرئيسة والتفاعلات بينها.

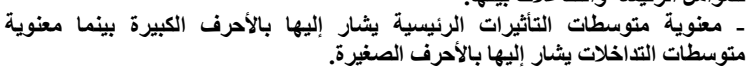

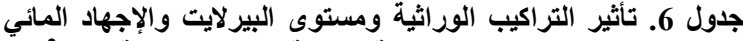

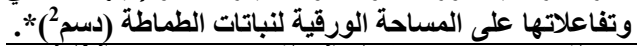

\begin{tabular}{|c|c|c|c|c|c|}
\hline 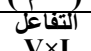 & \multicolumn{3}{|c|}{ مسنتويات البيزلايت } & \multirow{2}{*}{ مالريلات } & \multirow{2}{*}{ الورزاثيّية } \\
\hline$\frac{\mathbf{V} \times \mathbf{I}}{1509}$ & $\frac{\mathbf{P}_{2}}{1839}$ & $\frac{\mathbf{P}_{1}}{1524}$ & $\mathbf{P}_{\mathbf{0}}$ & & \\
\hline $\begin{array}{l}150.9 \\
\text { abc }\end{array}$ & $\begin{array}{c}183.9 \\
b\end{array}$ & $\begin{array}{c}152.4 \\
\mathrm{~d}\end{array}$ & $\begin{array}{c}116.5 \\
\text { ef }\end{array}$ & $\mathrm{I}_{1}$ & \\
\hline 157.2 & 201.1 & 164.4 & 106.1 & $\mathrm{~J}_{0}$ & \\
\hline $\begin{array}{c}\mathrm{ab} \\
1593\end{array}$ & 2099 & $\begin{array}{c}c \\
1497\end{array}$ & $\mathrm{fg}_{3}$ & $1_{2}$ & \\
\hline $\begin{array}{c}159.3 \\
\mathrm{a}\end{array}$ & 209.9 & $\mathrm{~d}$ & $\mathrm{e}^{110.5}$ & $\mathrm{I}_{1}$ & \\
\hline 153.2 & 179.1 & 185.6 & 94.9 & $\mathrm{I}_{2}$ & \\
\hline $\begin{array}{c}\mathrm{ab} \\
141.3\end{array}$ & $\begin{array}{c}\mathrm{b} \\
152.8\end{array}$ & $\begin{array}{c}\mathrm{b} \\
148.2\end{array}$ & $12 \frac{\mathrm{g}}{3} .0$ & 12 & \\
\hline$\stackrel{c}{c}$ & $\begin{array}{c}\mathrm{d} \\
184.0\end{array}$ & $\stackrel{d}{d}$ & $\stackrel{\mathrm{e}}{\mathrm{e}}$ & 11 & \\
\hline $\mathrm{bc}$ & $\begin{array}{c}\mathrm{b} \\
185.1 \\
\mathrm{~A}\end{array}$ & $\begin{array}{c}\mathrm{d} \\
158.6 \\
\mathrm{~B}\end{array}$ & $\begin{array}{c}\mathrm{fg} \\
110.8 \\
\mathrm{C}\end{array}$ & $1_{2}$ & \\
\hline & \multicolumn{5}{|c|}{ التفاعل بين التركيب الور انتي و البيرلايت } \\
\hline المتوسط & \multicolumn{3}{|c|}{ مسنتويات البيرلايت } & \multicolumn{2}{|c|}{ التراكيب الوراثية } \\
\hline 154.1 & 192.5 & 158.4 & 111.3 & & \\
\hline A & $\mathrm{a}$ & & & & \\
\hline 156.2 & 194.5 & 167.6 & 106.6 & & \\
\hline A & a & $\mathrm{b}$ & e & & \\
\hline 144.2 & 168.4 & 149.6 & 114.7 & & \\
\hline & \\
\hline المتو سط & \multicolumn{3}{|c|}{ مسنتويات البيرلايت } & & د ت الر \\
\hline 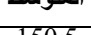 & $P_{2}$ & $\mathbf{P}_{1}$ & $\mathbf{P}_{0}$ & & \\
\hline 150.5 & 182.2 & 150.1 & 119.3 & & \\
\hline A & a & c & d & & \\
\hline $\begin{array}{c}152.5 \\
\mathrm{~A}\end{array}$ & $\begin{array}{c}188.1 \\
\mathrm{a}\end{array}$ & $\begin{array}{c}167.0 \\
\mathrm{~b}\end{array}$ & $\begin{array}{c}102.4 \\
\mathrm{e}\end{array}$ & & \\
\hline & & & & & \\
\hline
\end{tabular}

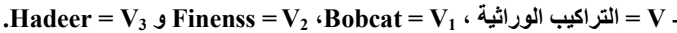

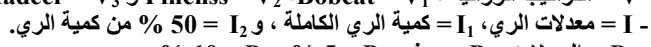

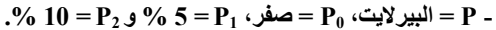

ـ ـ هناك فروق مغنوية بين المتوسطات التي تحمل حروفاً مختلفة عند مستوى احتمال 0.05 للعوامل الرئيسة والتفاعلات بينها.

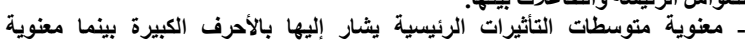
متوسطات التداخلات يشار إليها بالأحرف الصغيرة. 
Meléndez-Martínez, A. ; A. Nascimiento ; Y. Wang ; C. Liu ; Y. Mao and X. Wang. 2013. Effect of tomato extract supplementation against high-fat diet-induced hepatic lesions. Hepatobiliary Surgery and Nutrition. 2(4):198-208.

Nelson, P. V. 2012. Greenhouse Operation and Management. 7th ed. Pearson, Upper Saddle River, NJ.

Olle, M. 2016. The Effect of vermicompost based growth substrates on tomato growth. Journal of Agricultural Science.27(1):38-41.

Parameshwarareddy, R.; S.S. Angadi and M.S. Biradar. 2017. Effect of drip irrigation levels and substrates on growth, yield and quality of tomato under protected condition. The Bioscan. 12(1):447-452.

Perveen, R. ; H.A.R. Suleria ; F.M. Anjum ; M.S. Butt ; I. Pasha and S. Ahmad. 2015. Tomato (Solanum lycopersicum) carotenoids and lycopenes chemistry; metabolism, absorption, nutrition, and allied health claims - a comprehensive review. Critical Reviews in Food Science and Nutrition. 55:919-929.

Schmilewski, G. 2009. Growing medium constituents used in the eu. Acta Hortic. 819(1): 33-46.

Sedaghat, M.; H. Kazemzadeh-Beneh; M. Azizi and M. Momeni. 2017. Optimizing Growing Media for Enhancement to Vegetative Growth, Yield and Fruit Quality of Greenhouse Tomato Productionin Soilless Culture System. World Journal of Agricultural Sciences. 13(2):82-89.

Statistical Analysis System (SAS) Institute. 2008. The SAS System for Windows, Release 8.1. Stat Anal Syst Inst, Cary, NC, USA.

Steel, R.G.; J.H. Torrie. 1980. Principles and Procedures of Statistics: A Biometrical Approach. 2nd ed McGraw Hill Book Co, New York.

Verdonck, O. and P. Demeyer. 2004. The influence of the particle size on physical properties of growing media. Acta Hort. 644: 99-101.

Evans, M.R. 2004. Ground bovine bone as a perlite alternative in horticultural substrates. Hort. Technology 14:171-175.

Fajinmi, A. A. and O. B Fajinmi. 2010. An overview of bacterial wilt disease of tomato in Nigeria. Agricultural Journal. 5(4): 242-247.

Ghehsareh, A. M. ; S. Najmeh and B. Hasan . 2011. Comparison of date-palm wastes and perlite as growth substrates on some tomato growing indexes. African Journal of Biotechnology.10(24): 4871-4878.

Giuliani, M.M. ; E. Nardella ; G. Gatta ; A. De Caro and M. Quitadamo. 2011. Processing tomato cultivated under water deficit conditions: the effect of azoxystrobin. Acta Hort. 914:287-294.

Haddad, M. 2007. Effect of three substrates on growth, yield and quality of tomato by the use of geothermal water in the south of Tunisia. Journal of Food, Agriculture and Environment. 5(2): $175-178$.

Ibrahim, A. ; M. Wahb-Allah ; H. Abdel-Razzak and A. Alsadon. 2014. Growth, yield, quality and water use efficiency of grafted tomato plants grown in greenhouse under different irrigation levels. Life Science Journal. 11(2):118-126.

IPGRI. 1996. Desecriptors for tomato (Lycopersicon Sp.) Interational Plant Gentic Resources Institute, Rome, Italy. Pp. 44.

Mahadeen, A. ; O. Mohawesh ; K. Al-Absi and W. AlShareef. 2011. Effect of irrigation regimes and frequency on water use efficiency and tomato fruit (Lycopersicon esculentum Mill.) grown under an arid environment. Arch Agron Soil Sci. 57:105-114.

Mehadi, S. ; B. Mohammed and W. Yonas. 2016. Participatory variety selection of improved tomato (Lycopersicum esculentum Mill.) varieties in the lowlands of Bale, South-Eastern Ethiopia. African Journal of Agricultural Economics and Rural Development.4 (7):458462.

\section{Effect of Perlite on Reducing Water Stress for Three Genotypes of Tomatoes Solanum lycopersicum L.}

\section{Vegetative growth}

AL-Shammary, A. M. A. ${ }^{1}$; M. A. Abood ${ }^{2}$ and G. J. Hamdi ${ }^{1}$

${ }^{1}$ Prof. Dept. of Hort. and Landscape - College of Agric-Univ. of Diyala

${ }^{2}$ Assist. Prof.-Dept. of Soil and water Resources- College of Agric- Univ. of Diyala ghassanhamdi38@gmail.com

\section{ABSTRACT}

Experiment was conducted during spring season 2016 in the experimental station of the Department of Horticulture and Landscape, College of Agriculture Diyala University, Iraq. The aim of the study was to evaluate the effect of three factors, first factor: three tomato genotypes namely 'bobcat' $\left(\mathrm{V}_{1}\right)$, 'finenss' $\left(\mathrm{V}_{2}\right)$ and 'hadeer' $\left(\mathrm{V}_{3}\right)$; second factor: Irrigation levels, control treatment $(100 \%)$ depletion of $50 \%$ of field capacity and $50 \%$ of the amount water of the control. Three levels of perlite $\left(\mathrm{P}_{0}, 5\right.$ $\%$ and $10 \%$ ) as a volumetric percentage of soil. The experiments were arranged in a Split-Split Plot Design in R.C.B.D. The study results showed Finenss genotype had significant superiority in Plant height and leaves number per plant $(103.87 \mathrm{~cm}$ and 78.39 leaf plant $\left.^{-1}\right)$. While irrigate plants with $50 \%$ irrigation $\left(I_{2}\right)$ gave the highest leaves number (63.15 leaf per plant). While plants treated with $10 \%$ perlite had superiority in plant height, stem diameter, total branches number, leaves number, total leaf area plant results were, $89.30 \mathrm{~cm}, 1.64 \mathrm{~cm}, 29.33$ branches plant $^{-1}, 65.89$ leaf plant ${ }^{-1}, 185.13 \mathrm{dc}$ plant $^{-1}$ respectively. Superiority transactions morally $\mathrm{V}_{2} \mathrm{I}_{2} \mathrm{P}_{2}$ had superiority in plant length, branches number and leaves number $(114.20 \mathrm{~cm}, 35.33$ branches plant $^{-1}, 95.67$ leaf plant $^{-1}$ ) respectively. $\mathrm{V}_{2} \mathrm{I}_{1} \mathrm{P}_{2}$ While treatments superiority significant in stem diameter and total leaves area in plant $\left(1.80 \mathrm{~cm}\right.$ and $209.86 \mathrm{dc}^{2}$ plant $\left.^{-1}\right)$ respectively.

Keywords: genotypes, perlite, water stress, tomato 